\title{
A case report of postural tachycardia syndrome after COVID-19
}

\author{
Mitchell G. Miglis $^{1} \cdot$ Thomas Prieto $^{1} \cdot$ Ruba Shaik $^{1} \cdot$ Srikanth Muppidi ${ }^{1} \cdot$ Dong-In Sinn ${ }^{1} \cdot$ Safwan Jaradeh $^{1}$
}

Received: 4 August 2020 / Accepted: 28 August 2020 / Published online: 3 September 2020

(c) Springer-Verlag GmbH Germany, part of Springer Nature 2020

\section{Dear Editors,}

The most common symptoms of coronavirus disease 19 (COVID-19) from severe acute respiratory syndrome coronavirus 2 (SARS-CoV-2) infection include fever, cough, sore throat, and fatigue. As case numbers grow, neurological symptoms have been reported with increasing frequency, including those of autonomic dysfunction [1]. Most neurological reports detail symptoms in hospitalized patients during the para-infectious period; thus, understanding of longer-term, post-infectious sequalae is limited. We report the case of a patient who developed postural tachycardia syndrome (POTS) several months after confirmed SARSCoV-2 infection.

In early March of 2020, a 26-year-old emergency department nurse in Orange County, California developed a mild cough and an itchy throat. She woke the next day with palpitations, fatigue, and mild shortness of breath. On day 3 she presented to urgent care and had a nasopharyngeal swab which returned negative for SARS-CoV-2 PCR. On day 7 she woke up with palpitations, shortness of breath, and anorexia. She noted that her resting seated HR was $110 \mathrm{bpm}$ and would increase to $190 \mathrm{bpm}$ after walking up a flight of stairs. Her cough worsened and she developed burning chest pains on inhalation. She presented to the emergency department for evaluation, where her temperature was $100.4^{\circ} \mathrm{F}$ and her $\mathrm{SaO} 298 \%$. A chest CT was performed and demonstrated moderate bronchitis with right lower lobe atelectasis. Her SARS-CoV nasopharyngeal swab was repeated and returned positive. IgG and IgM antibodies to the SARS-CoV-2 spike

Electronic supplementary material The online version of this article (https://doi.org/10.1007/s10286-020-00727-9) contains supplementary material, which is available to authorized users.

Mitchell G. Miglis

mmiglis@stanford.edu

1 Department of Neurology and Neurological Science, Autonomic Division, Stanford University, 213 Quarry Road, Palo Alto, CA 94304, USA receptor binding domain (RBD) also returned positive. She was given IV fluids, a 5-day prescription of azithromycin, and discharged home.

Over the following week she noted continued tachycardia (standing HR of $150 \mathrm{bpm}$ after showering), chest pains, shortness of breath, fatigue and exercise intolerance, along with subjective fevers and insomnia. Her seated, resting BP increased to $156 / 112 \mathrm{mmHg}$ (her typical seated, resting BP was $110 / 60 \mathrm{mmHg}$ ). On day 19 she developed orthostatic lightheadedness and presyncope. She returned to the emergency department for evaluation, where her seated BP was 146/100 mmHg, HR was $125 \mathrm{bpm}$, and $\mathrm{SaO} 2$ dropped from $98 \%$ to $94 \%$ with minimal exertion. She was given IV fluids, kept overnight, and discharged the following day.

On day 22 she developed symptoms of hyperactivity with pressured speech and a feeling of inner restlessness. On day 24 she developed episodic sensations of "adrenaline surges" characterized by diarrhea, tremors, and worsening restlessness. Over the following month her orthostatic intolerance worsened, despite ongoing ability to exercise. Increased fluid and salt intake and compression garments were utilized, with minimal improvement in symptoms. On day 45 she developed episodic facial flushing, dermatographia, and non-pruritic hives. She presented to our autonomic clinic for evaluation at this time.

Her past medical history included exercise-induced asthma and obsessive-compulsive disorder, diagnosed at age 16, which resolved without pharmacological treatment by age 18 . She had no history of autonomic symptoms or syncope. Her family history was notable for similar symptoms in her twin sister, a close contact, who experienced a similar infectious prodrome during the same time period; however, her sister tested negative for SARS-CoV-2, twice, by the same lab that analyzed the patient's samples. Physical examination of our patient, including a detailed neurological examination, was normal. There was no evidence of joint hypermobility. We prescribed propranolol and clonidine which led to some improvement of her hyperadrenergic symptoms. 
Despite these medications, her symptoms of orthostatic intolerance and spells of sympathetic hyperactivity continued, and on day 107, over 3 months after symptom onset, she underwent autonomic reflex testing in our laboratory, including quantitative sudomotor axon reflex testing (QSART), heart rate variability with deep breathing, Valsalva maneuver, and a 10-min head-up tilt (HUT) at an angle of $70^{\circ}$. Beat-to-beat BP was measured with finger plethysmography and confirmed with an automated cuff sphygmomanometer over the brachial artery. An intravenous catheter was placed, and supine and upright norepinephrine (NE) levels were collected. All medications were held 1 week prior to testing.

Autonomic testing demonstrated an exaggerated postural tachycardia with an HR increase of $65 \mathrm{bpm}$ on HUT, episodic hypertensive systolic BP surges to $170 \mathrm{mmHg}$ in an oscillatory pattern, and robust BP responses to Valsalva maneuver pattern in the absence of hyperventilation, suggestive of a hyperadrenergic state (Fig. 1). The heart rate response to HUT demonstrated a sustained increase of greater than $30 \mathrm{bpm}$ which persisted for the duration of the tilt, arguing against an anxiety response. The remainder of her autonomic testing and NE levels were normal (supplementary table). Her nasopharyngeal swab was repeated and was negative for SARS-CoV-2. IgM and IgG RBD antibodies were repeated and were also negative, as were ganglionic acetylcholine receptor antibodies. At the time of this report, approximately 5.5 months after symptom onset, the patient's symptoms remain, and she has not yet returned to work.

We report the first case, to our knowledge, of POTS developing after SARS-CoV-2 infection. Our patient developed tachycardia along with common respiratory symptoms of COVID-19 in the acute phase of her illness; however, these symptoms resolved within 3 weeks, while her tachycardia and other symptoms of autonomic impairment persisted and intensified over the following several months. Hyperadrenergic symptoms were a prominent feature in our patient, and her autonomic testing demonstrated features often reported in hyperadrenergic POTS; however, her serum NE levels returned normal, and in fact lower than typically reported in POTS, which deserves further investigation in future cases. It should also be noted that our patient's symptoms have now persisted for 5.5 months, and some definitions of POTS require a symptom duration of at least 6 months.

Infection is a well-described trigger of POTS, with $28-41 \%$ of patients reporting onset after a viral prodrome [2, 3]. In addition, autonomic dysfunction has been described in small cohorts after the 2002 SARS epidemic [4], and several other pathogens have been associated with autonomic dysfunction [5]. While an immune-mediated mechanism has been suggested, the pathophysiology remains to be elucidated. It is worth noting that our patient's antibodies were initially positive on day 7 but were undetectable 2 months later, as reported in other patients with COVID-19. While our patient had no pre-existing symptoms of autonomic impairment, it is worth noting that her twin sister also developed similar symptoms after a similar infectious prodrome. This reinforces the importance of phenotyping to better understand vulnerability to both the severe acute manifestations and the chronic sequalae of COVID-19. Given the magnitude of the pandemic, COVID-19 represents a unique opportunity to study post-infectious autonomic syndromes,
$1 \mathrm{~A}$
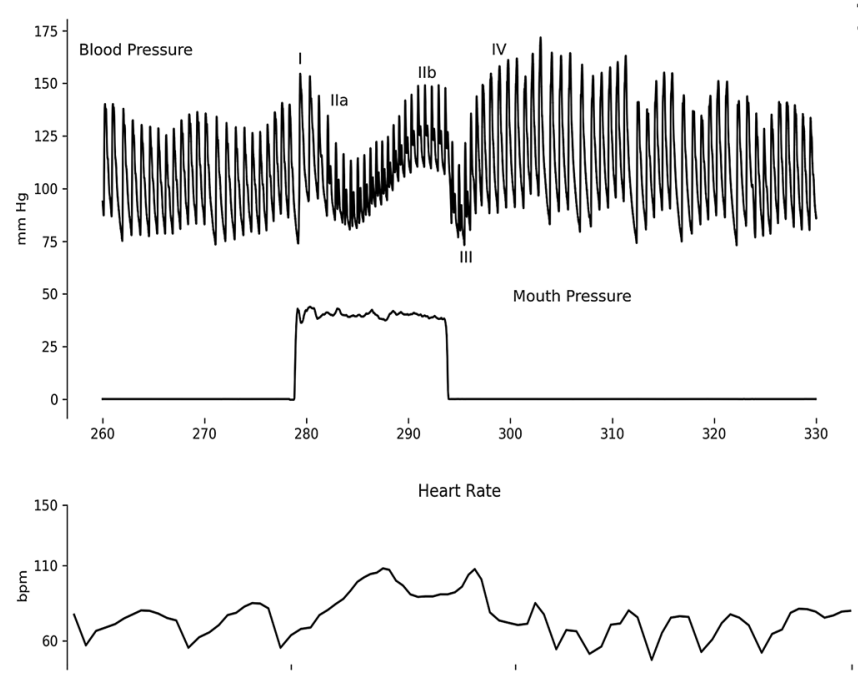

1B

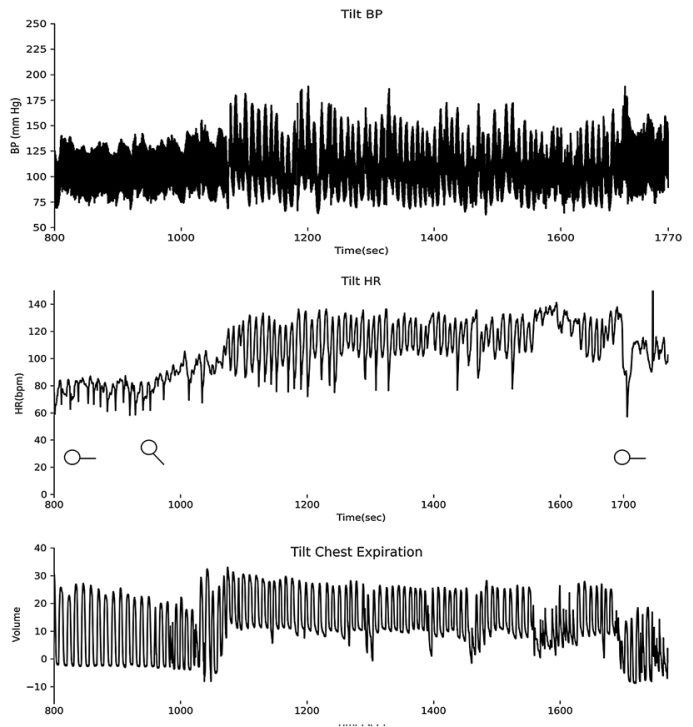

Fig. 1 a Valsalva maneuver demonstrating a robust BP and HR response. b Head-up tilt testing at $70^{\circ}$ demonstrating an exaggerated postural tachycardia with an HR increase of $65 \mathrm{bpm}$ and epi- sodic hypertensive systolic BP surges in an oscillatory pattern in the absence of hyperventilation 
research which will hopefully lead to greater understanding of the pathogenic mechanisms of POTS.

Author contributions MM: drafting and revision of manuscript; TP, RS, SM, DS, SJ: revision of manuscript.

\section{Funding None.}

Data availability Autonomic testing data available on request.

\section{Compliance with ethical standards}

Conflicts of interest On behalf of all authors, the corresponding author states that there is no conflict of interest.

Informed consent The patient has given verbal consent to publication of this case.

\section{References}

1. Romero-Sánchez CM, Díaz-Maroto I, Fernández-Díaz E et al (2020) Neurologic manifestations in hospitalized patients with COVID-19: The ALBACOVID registry. Neurology. https://doi. org/10.1212/WNL.0000000000009937.10.1212/wnl.0000000000 009937

2. Thieben MJ, Sandroni P, Sletten DM et al (2007) Postural orthostatic tachycardia syndrome: the Mayo Clinic experience. Mayo Clin Proc 82:308-313. https://doi.org/10.4065/82.3.308

3. Shaw BH, Stiles LE, Bourne K et al (2019) The face of postural tachycardia syndrome - insights from a large cross-sectional online community-based survey. J Intern Med 286:438-448. https ://doi.org/10.1111/joim.12895

4. Lo YL, Leong HN, Hsu LY et al (2005) Autonomic dysfunction in recovered severe acute respiratory syndrome patients. Can J Neurol Sci 32:264

5. Carod-Artal FJ (2018) Infectious diseases causing autonomic dysfunction. Clin Auton Res 28:67-81 